\title{
Coronary Spastic Angina Causing Myocardial Stunning Associated with Reversible Wall Thinning and Motion Abnormality of the Left Ventricle: a Case Report
}

\author{
Toshiaki Isogai, Hiroyuki Tanaka, Motohiro Asaki, Tetsuro Ueda
}

\begin{abstract}
We describe a rare case of coronary spastic angina (CSA) causing myocardial stunning with reversible wall thinning and motion abnormality of the left ventricle (LV). A 70-year-old woman presented with sudden resting angina. A 12-lead electrocardiogram showed typical ST-T changes of acute myocardial infarction. Although left ventriculography revealed akinesis from the apex to the mid-anterior LV, urgent coronary angiography did not show an obstructed coronary artery. Cardiac magnetic resonance imaging (MRI) identified thinning of the anterior wall in the akinetic region, without any late gadolinium enhancement. Cardiac single photon emission computed tomography (SPECT), using ${ }^{123}$ iodine beta methyl-iodophenyl pentadecanoic acid ( ${ }^{123}$-BMIPP) and ${ }^{201}$ thallium $\left({ }^{201} \mathrm{TI}\right)$, showed a mismatch congruent with the akinetic region. An acetylcholine provocation test performed during hospitalization revealed multivessel coronary spasms in all 3 epicardial arteries. After initiation of treatment with coronary vasodilators, the wall thinning and motion abnormality gradually recovered to the normal range. In the present case, CSA caused myocardial stunning associated with reversible wall thinning and motion abnormality in the acute phase. CSA can occasionally lead to two conditions associated with LV wall abnormalities: myocardial infarction and stunning. Although the findings of the 2 conditions are similar in the acute phase, stunning can be differentiated from infarction using multiple imaging modalities. Cardiac MRI and SPECT are useful tools for determining whether the myocardium is infarcted or stunned.

KEY WORDS: coronary spastic angina, myocardial stunning, myocardial infarction, magnetic resonance imaging, single photon emission computed tomography
\end{abstract}

\section{Case Presentation}

A 70-year-old woman presented to our emergency department with sudden resting angina at 8 p.m. Although she had experienced similar episodes over the previous 2 years, she had not previously sought medical attention, as these episodes had not been severe. She was a non-smoker with a prior history of untreated hypertension. On admission, her blood pressure was high (systolic/diastolic blood pressure, 173/109 $\mathrm{mmHg}$ ), but findings of physical examination were otherwise unremarkable. A 12 lead electrocardiogram (ECG) showed ST-segment elevation in leads I, aVL, and V2-V6 and reciprocal ST-segment depression in leads II, III, and aVF (Fig. 1A). The symptoms were resolved with an intravenous injection of isosorbide dinitrate. A transthoracic echocardiogram demonstrated akinesis in the region from the apex to the mid-anterior left ventricle (LV). Urgent coronary angiography did not demonstrate an obstructed coronary artery (Fig. 2, upper images). However, left ventriculography (LVG)

Department of Cardiology, Tokyo Metropolitan Tama Medical Center, 2-8-29 Musashidai, Fuchu, Tokyo 183-8524, Japan

Received June 28, 2013; Accepted September 30, 2013

doi: 10.7793/jcoron.19.13-00006 revealed akinesis in the same region as did the echocardiogram (Fig. 3).

The patient's blood samples showed slightly elevated levels of cardiac troponin T (50-100 ng/l; normal range, 0-50 ng/l), slightly elevated creatine kinase (CK, 202 IU/l; normal range, 30-150 IU/1; maximum CK during hospitalization, 269 IU/1), and increased heart-type fatty acid-binding protein $(14.2 \mathrm{ng} / \mathrm{ml}$; normal range, 0-6.2 ng/ml), but CK-MB remained within normal limits (15 IU/1; normal range, 0-25 IU/1; maximum CK-MB during hospitalization, $20 \mathrm{IU} / \mathrm{l})$.

To investigate the myocardial damage in detail, we performed cardiac magnetic resonance imaging (MRI) on hospitalization day 2. Although cardiac MRI showed anterior wall thinning, late gadolinium enhancement (LGE) was not detected (Fig. 4). We also examined the cardiac single photon emission computed tomography (SPECT) using ${ }^{123}$ iodine beta methyl-iodophenyl pentadecanoic acid ( $\left.{ }^{123} \mathrm{I}-\mathrm{BMIPP}\right)$ and ${ }^{201}$ thallium $\left({ }^{201} \mathrm{Tl}\right)$ on hospitalization day 5. The dual cardiac SPECT revealed a mismatch from the apex to the mid-anterior LV; ${ }^{123}$ I-BMIPP images showed that the defect was ischemic memory (Fig. 5). Thus, cardiac MRI and SPECT were able to differentiate between infarcted and stunned myocardium. 


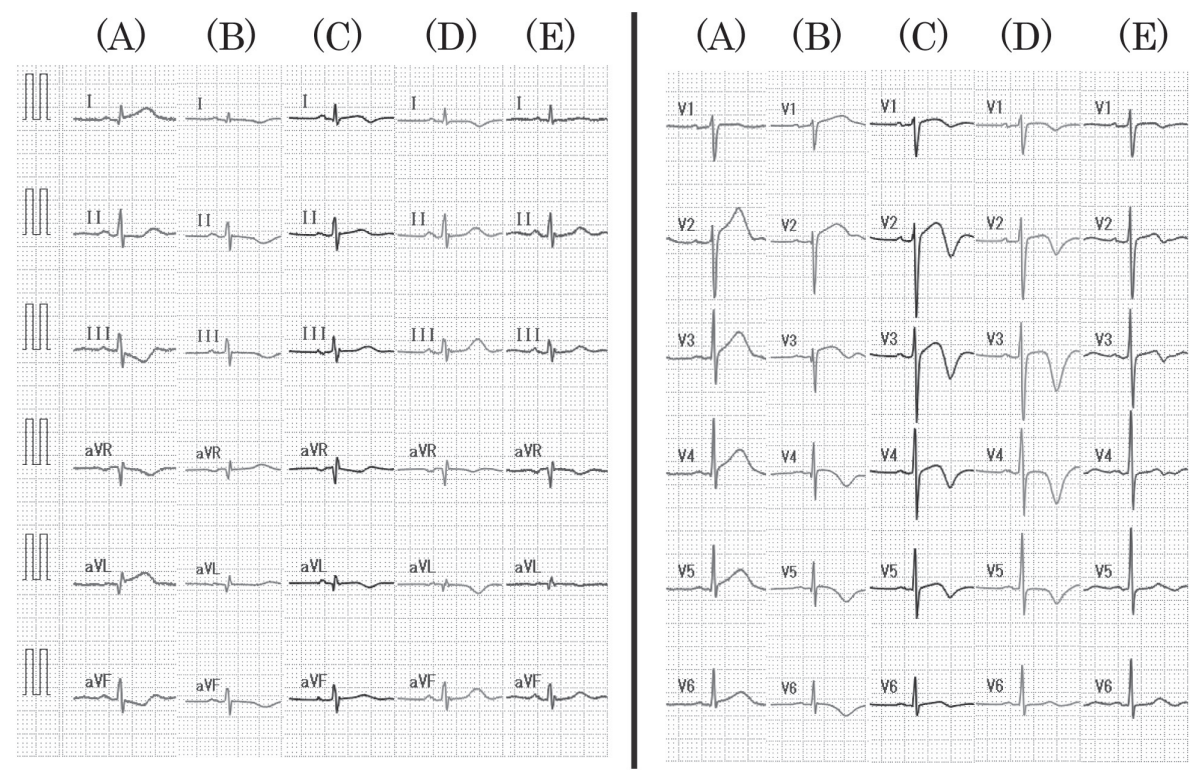

Fig. 1 Twelve-lead electrocardiographic findings.

The traces were obtained (A) on admission, (B) on hospitalization day 2, (C) at the time of discharge (18 days after admission), (D) at 2 weeks after discharge (E) at 6 months after discharge.

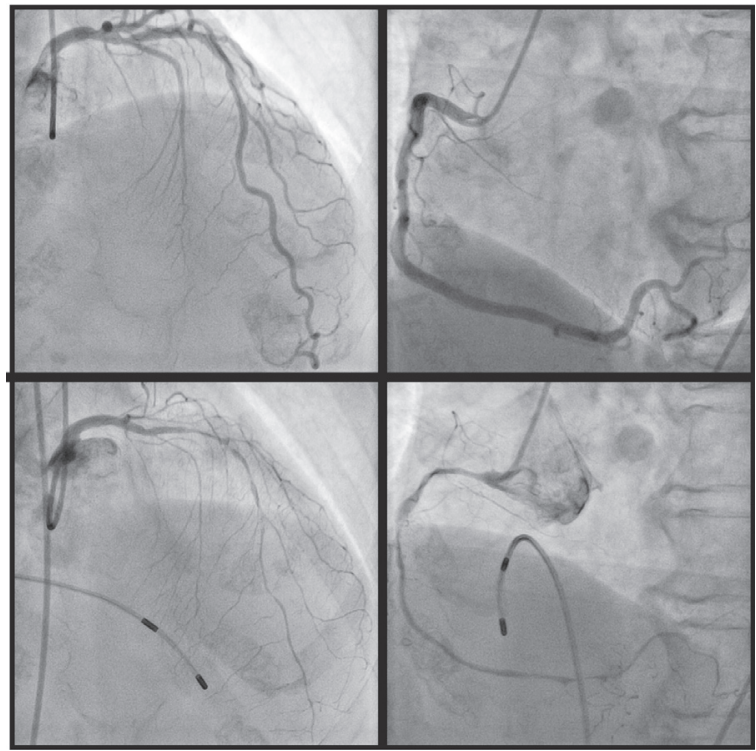

Fig. 2 Coronary angiography (CAG)

Left: left coronary artery, right anterior oblique (RAO) view. Right: right coronary artery. RAO view.

Urgent CAG post-admission (upper images) did not demonstrate an obstructed coronary artery. The elective CAG, with intra-coronary acetylcholine $(20 \mu \mathrm{g})$ injection into each coronary artery (lower images), demonstrated multivessel coronary spasms in the 3 epicardial arteries.

To determine the cause of the patient's resting angina, an acetylcholine provocation test was performed on hospitalization day 7. The test revealed multivessel coronary spasms in all 3 epicardial arteries (segment 7, 99\% with delay; segment 14, 99\%; segment 4AV, 99\% with delay) in conjunction with her chest symptoms (Fig. 2, lower images). Therefore, we diagnosed her with

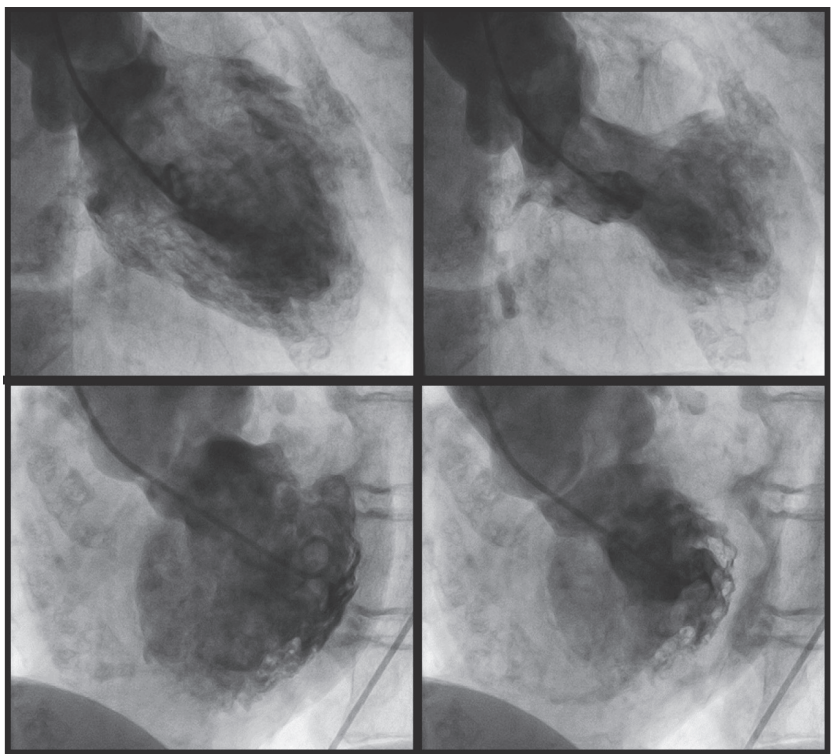

Fig. 3 Left ventriculography (LVG). Left: end-diastole; Right: end-systole.

Upper: right anterior oblique view; Lower: left anterior oblique view. On admission, LVG showed akinesis from the apex to the midanterior wall, with hypokinesis in the interventricular septum and lateral wall.

coronary spastic angina (CSA). The patient began taking a calcium channel blocker (benidipine, $8 \mathrm{mg} /$ day) and a potassium channel opener (nicorandil, $30 \mathrm{mg} /$ day), and her chest symptoms did not reappear.

Over the course of hospitalization, the patient's ECGs changed as shown in Fig. 1. The leads with ST-segment elevation showed T-wave inversion on hospitalization day 2. Subsequently, T- 

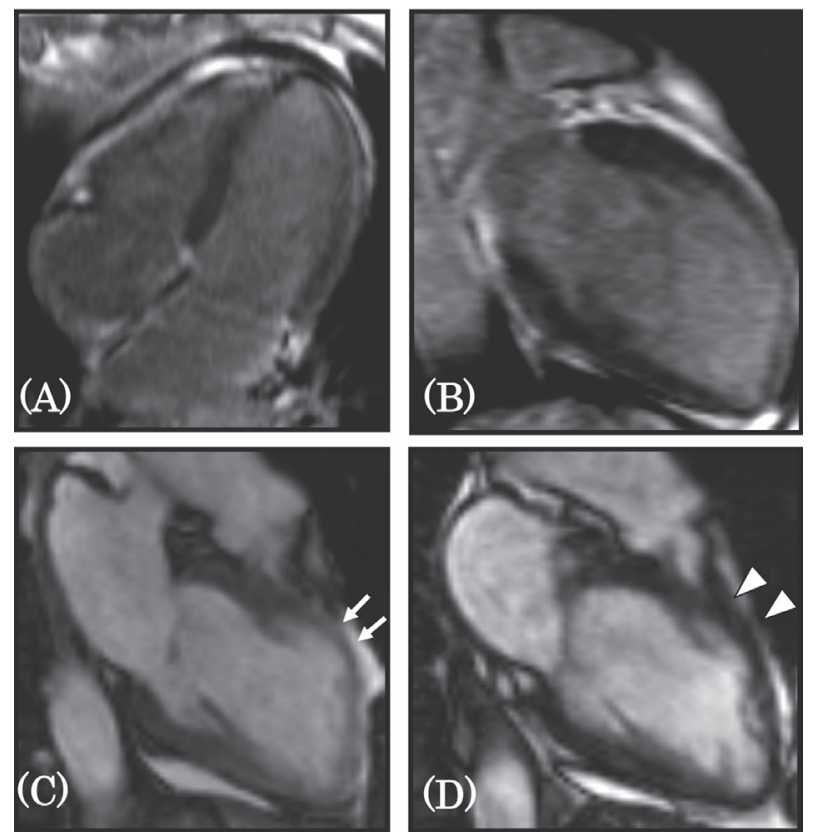

Fig. 4 Cardiac magnetic resonance imaging (MRI).

Late gadolinium-enhancement images, (A) 4-chamber view, (B) vertical long axial view. Cine MRI images, (C) and (D): vertical long axial view. Cardiac MRI performed on the second day of hospitalization did not demonstrate late gadolinium enhancement (A, $\mathrm{B})$; anterior wall thinning was identified (C, arrows). Cardiac MRI performed 6 months later revealed recovery of wall thickness (D, arrowheads) and wall motion.

waves with QT prolongation appeared deeper, especially in the precordial leads and persisted over 2 months. The T-waves slowly recovered, but the terminal $\mathrm{T}$-waves remained slightly inverted 6 months after discharge.

The LV ejection fraction, as evaluated by echocardiography, gradually recovered during the patient's hospitalization. On postadmission day 18 , she was discharged without any symptoms. A 6-month post-discharge cardiac MRI showed full recovery of LV wall thickness and motion (Fig. 4). On the basis of these results, we concluded that CSA caused myocardial stunning associated with reversible wall thinning and wall motion abnormality of the $\mathrm{LV}$ without myocardial infarction.

\section{Discussion}

We treated a rare case of CSA that produced a reversible wall abnormality. Upon admission, the initial clinical suspicion was that myocardial infarction caused sudden resting angina and LV abnormality because of the typical episodes and ECG changes. However, urgent coronary angiography failed to show obstructed coronary arteries. During the acute phase, a cardiac MRI did not detect LGE, despite the wall abnormality. Cardiac SPECT also showed metabolic changes congruent with the damaged region. As a result, the clinical condition was determined to be myocardial stunning rather than infarction.

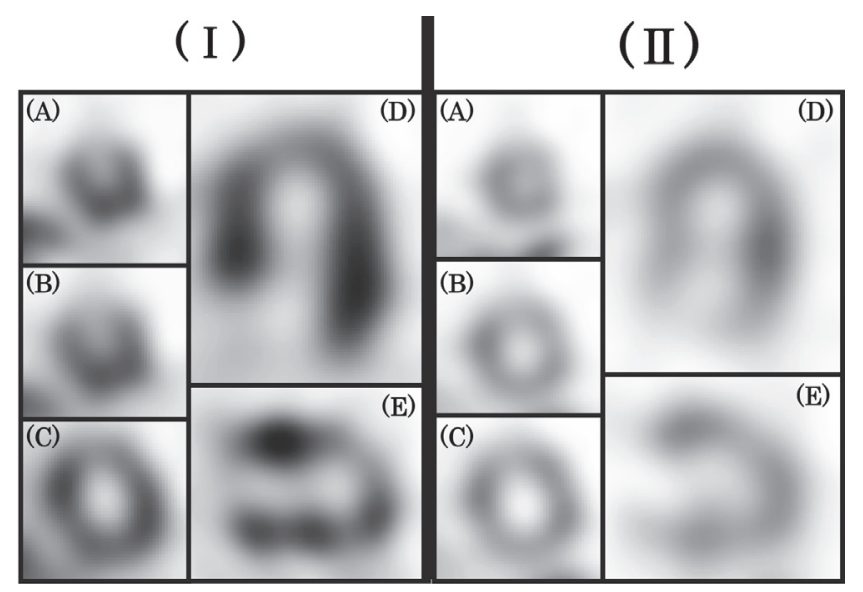

Fig. 5 Cardiac single photon emission computed tomography (SPECT) using ${ }^{123}$ iodine beta methyl-iodophenyl pentadecanoic acid $\left({ }^{123} \mathrm{I}\right.$-BMIPP) and ${ }^{201}$ thallium $\left({ }^{201} \mathrm{Tl}\right)$.

Images obtained using ${ }^{123} \mathrm{I}-\mathrm{BMIPP}$ : (I) and ${ }^{201} \mathrm{Tl}$ : (II). (A-C): short axial view; (D): vertical long axial view; (E): horizontal long axial view. Dual cardiac SPECT revealed a mismatch from the apex to the mid-anterior left ventricle because the ${ }^{123}$ I-BMIPP images showed the defect as ischemic memory.

Myocardial stunning is a post-ischemic mechanical dysfunction that persists after reperfusion despite the absence of irreversible cell necrosis. ${ }^{1-3)}$ The benefits of reperfusion therapy might be delayed, but occur within hours to days after reperfusion. Consequently, full recovery might be delayed for some weeks or months., ${ }^{4)}$ The diagnosis of myocardial stunning ${ }^{6)}$ requires evidence that (1) the contractile abnormality is reversible, with preservation of cardiac viability, and (2) the dysfunctional myocardium retains normal or near-normal flow. Myocardial stunning is caused by organic stenosis or coronary artery spasms, both of which are associated with severe, acute ischemia.

Prinzmetal et al first described CSA as a variant of angina in 1959. ${ }^{7}$ CSA is caused by episodic coronary artery spasms and is associated with transient ST-segment elevation. Each spasm is a reversible stenosis that limits coronary blood flow under resting conditions, occasionally causing myocardial infarction or stunning in cases of severe and prolonged ischemia. ${ }^{8,9)}$ Because both myocardial infarction and stunning show similar chest symptoms, elevated cardiac markers, ECG findings, and LV wall abnormalities, cardiac MRI and SPECT are used to differentiate between the 2 conditions in our hospital.

Cardiac MRI with a gadolinium-based contrast agent offers high spatial resolution and can identify myocardial infarction or fibrosis. ${ }^{10-12)}$ A previous study showed that LGE in cardiac MRI has a higher sensitivity and specificity for determining infarction than does SPECT. ${ }^{13)}$ Despite the presence of myocardial stunning, myocardial injury without necrosis does not show LGE. In addition, cardiac MRIs clearly demonstrate mechanical changes in the heart. ${ }^{14)}$ In our hospital, a patient with suspected myocar- 
dial infarction or stunning who demonstrates LGE in the transmural or subendocardial area covered by a stenotic coronary artery is diagnosed with myocardial infarction. Cardiac SPECT is useful to demonstrate myocardial stunning. ${ }^{15)}$ At-rest imaging with ${ }^{123} \mathrm{I}-$ BMIPP after an ischemic episode can identify regions of suppressed fatty acid metabolism as ischemic memory. ${ }^{16)}$ Despite restoration of blood flow, as identified by at-rest imaging with ${ }^{201} \mathrm{Tl}$, prolonged and persistent disturbances in fatty acid metabolism represent a scintigraphic marker of myocardial stunning.

In the present case, the patient's heart was not infarcted, but stunning was evident based on her cardiac MRI and SPECT results. Subsequently, since she had experienced similar episodes of resting angina before her present admission, we performed an acetylcholine provocation test to determine the cause of her myocardial stunning. Results of this test showed that CSA caused her angina and severe ischemia on admission, subsequently leading to myocardial stunning. To prevent an angina attack or recurrent myocardial stunning, medical therapy for the CSA is critical.

Before reaching this diagnosis, we also considered the possibility that her clinical condition was a type of takotsubo cardiomyopathy (TC) triggered by physical and emotional stress, and associated with CSA. However, we diagnosed her with CSA because (1) the reciprocal changes observed in her ECG on admission are infrequently observed in TC cases, ${ }^{17,18)}$ (2) CSA was identified as the cause of her resting angina, and (3) the LV wall motion in the acute phase did not show the typical TC apical ballooning pattern. Nevertheless, we could not rule out a diagnosis of TC because the mechanisms underlying TC remain unknown, and TC may account for some aspects of myocardial stunning.

In conclusion, the present case was interesting and instructive in demonstrating mechanical and metabolic changes associated with myocardial stunning through the use of cardiac MRI and SPECT. Myocardial stunning is difficult to differentiate from myocardial infarction in the acute phase, because of the similarities between the findings in the 2 conditions. However, differentiation should be attempted by using multiple imaging modalities. As demonstrated in the present case, cardiac MRI and SPECT are useful tools for achieving this differentiation and determining whether the myocardium is infarcted or stunned.

\section{References}

1) Bolli R: Mechanism of myocardial "stunning". Circulation 1990; 82: 723-738

2) Heyndrickx GR, Millard RW, McRitchie RJ, et al: Regional myocardial functional and electrophysiological alterations after brief coronary artery occlusion in conscious dogs. J Clin Invest 1975; 56: 978-985

3) Braunwald E, Kloner RA: The stunned myocardium: prolonged, postischemic ventricular dysfunction. Circulation 1982; 66: 1146-1149

4) Bolli R, Zhu WX, Thornby JI, et al: Time course and determinants of recovery of function after reversible ischemia in conscious dogs. Am J Physiol 1988; 254: H102-H114

5) Charlat ML, O'Neill PG, Hartley CJ, et al: Prolonged abnormalities of left ventricular diastolic wall thinning in the "stunned" myocardium in conscious dogs: time course and relation to systolic function. J Am Coll Cardiol 1989; 13: 185-194

6) Bolli R: Myocardial 'stunning' in man. Circulation 1992; 86: 1671-1691

7) Prinzmetal M, Kennamer R, Merliss R, et al: Angina pectoris. I. A variant form of angina pectoris; preliminary report. Am J Med 1959; 27: 375-388

8) Kishida H, Hata N, Kusama Y, et al: Factors influencing the clinical course and the long-term prognosis of patients with variant angina. Jpn Heart J 1987; 28: 293-306

9) Lambert CR, Pepine CJ: Coronary artery spasm and acute myocardial infarction. Cardiovasc Clin 1989; 20: 131-140

10) Wagner A, Mahrholdt H, Holly TA, et al: Contrast-enhanced MRI and routine single photon emission computed tomography (SPECT) perfusion imaging for detection of subendocardial myocardial infarcts: an imaging study. Lancet 2003; 361: 374 379

11) Kim RJ, Fieno DS, Parrish TB, et al: Relationship of MRI delayed contrast enhancement to irreversible injury, infarct age, and contractile function. Circulation 1999; 100: 1992-2002

12) Kim RJ, Wu E, Rafael A, et al: The use of contrast-enhanced magnetic resonance imaging to identify reversible myocardial dysfunction. N Engl J Med 2000; 343: 1445-1453

13) Kitagawa K, Sakuma H, Hirano T, et al: Acute myocardial infarction: myocardial viability assessment in patients early thereafter comparison of contrast-enhanced MR imaging with resting (201)Tl SPECT. Single photon emission computed tomography. Radiology 2003; 226: 138-144

14) John AS, Dreyfus GD, Pennell DJ: Images in cardiovascular medicine. Reversible wall thinning in hibernation predicted by cardiovascular magnetic resonance. Circulation 2005; 111: e24-e25

15) Hosokawa R, Nohara R, Fujibayashi Y, et al: Myocardial kinetics of iodine-123-BMIPP in canine myocardium after regional ischemia and reperfusion: implications for clinical SPECT. J Nucl Med 1997; 38: 1857-1863

16) Dilsizian V, Bateman TM, Bergmann SR, et al: Metabolic imaging with beta-methyl-p-[(123)I]-iodophenyl-pentadecanoic acid identifies ischemic memory after demand ischemia. Circulation 2005; 112: 2169-2174

17) Ogura R, Hiasa $Y$, Takahashi $T$, et al: Specific findings of the standard 12-lead ECG in patients with 'Takotsubo' cardiomyopathy: comparison with the findings of acute anterior myocardial infarction. Circ J 2003; 67: 687-690

18) Kosuge M, Ebina T, Hibi K, et al: Simple and accurate electrocardiographic criteria to differentiate takotsubo cardiomyopathy from anterior acute myocardial infarction. J Am Coll Cardiol 2010; 55: 2514-2516 